\title{
Agregação de um planossolo sistematizado há um ano e sob cultivo de arroz irrigado
}

\author{
Aggregation of an albaqualf under land leveling for one year and under flooded rice
}

\author{
José Miguel Reichert ${ }^{1}$ Cláudia Liane Rodrigues de Lima ${ }^{2}$ Ricardo Simão Diniz Dalmolin ${ }^{3}$ \\ Dalvan José Reinert ${ }^{1}$ Celso Gonçalves ${ }^{4}$ Marcelo Nunes $^{5}$
}

\section{RESUMO}

A sistematização do solo altera a variação horizontal e a vertical das propriedades do solo. O objetivo deste trabalho foi avaliar a influência das propriedades físicas e químicas do solo sobre o seu grau de agregação e verificar a semelhança de características relacionadas à agregação de horizontes do solo original e sistematizado (áreas de corte e aterro). Para a quantificação das propriedades intrínsecas $e$ do grau de agregação, amostras com estrutura alterada e preservada foram coletadas em dois perfis com características originais e em dois perfis localizados em áreas sistematizadas de um Planossolo hidromórfico eutrófico típico, perfazendo um total de seis perfis analisados. Verificou-se que a agregação do solo sistematizado (áreas de corte e aterro) foi, em geral, similar à dos horizontes $E$ e $B$ do perfil original do solo. A argila, os óxidos de ferro, alumínio e manganês, além do magnésio e do alumínio trocáveis, foram os principais componentes que se correlacionaram com a agregação do solo. Pela análise de regressão múltipla, verificou-se que houve relação positiva da agregação do solo com a argila e com os óxidos de ferro e de alumínio cristalinos e de baixa cristalinidade.

Palavras-chave: solos de várzea, sistemas de manejo, arroz, estrutura do solo.

\section{ABSTRACT}

Land leveling modifies horizontal and vertical soil properties variability. The objective of this work was to evaluate the influence of soil properties (physical and chemical) on aggregation and test the similarity on aggregation of original soil horizons and land leveling. To quantify the intrinsic properties and soil aggregation, disturbed and undisturbed soil samples were collected in two profiles with original characteristics and land leveling areas of an Albaqualf. Aggregation of land leveling was similar to $E$ and $B$ horizons of the original soil. The clay, iron, aluminum and manganese oxides besides the magnesium and exchangeable aluminum were main components that were correlated with soil aggregation Multiple regression analysis revealed positive relationship of the soil aggregation with clay and iron and aluminum oxide crystalline and low crystalline.

Key words: lowland, tillage systems, rice, soil structure.

\section{INTRODUÇÃO}

Os solos de várzea ocupam uma área de aproximadamente 5,4 milhões de hectares no RS, o que representa 20\% da área total do Estado. Esses solos são normalmente encontrados nas planícies de rios e lagos e formaram-se em condições de deficiência de drenagem (hidromorfismo), em conseqüência do relevo plano. Predominam cores acinzentadas ou escurecidas, indicativas de baixa permeabilidade.

Os solos de várzea normalmente estão sujeitos à saturação por água ou a alagamentos periódicos, condição esta favorável ao estabelecimento da cultura do arroz irrigado. No estado, a lavoura de arroz participa com $40 \%$ da produção de grãos, ocupando uma área de 950 mil hectares, o que representa 6,8\% da produção de grãos do país (AZAMBUJA et al., 2004).

\footnotetext{
${ }^{1}$ Departamento de Solos, Centro de Ciências Rurais (CCR), Universidade Federal de Santa Maria (UFSM), 97105-900, Santa Maria, RS, Brasil. E-mail: reichert@smail.ufsm.br; reinert@ccr.ufsm.br.

${ }^{2}$ Departamento de Solos, CCR, UFSM, Santa Maria, RS, Brasil. E-mail: clrlima@yahoo.com.br. Autor para correspondência.

${ }^{3}$ Departamento de Solos, CCR, UFSM, Santa Maria, RS, Brasil. E-mail: dalmolin@ccr.ufsm.br.

${ }^{4}$ Departamento de Solos, CCR, UFSM, Santa Maria, RS, Brasil. E-mail: celso@mail.ufsm.br.

${ }^{5}$ Departamento de Geoquímica, Universidade de Aveiro (UA), Aveiro, Portugal. E-mail:mnunes@geo.ua.pt.
} 
Dentre as principais classes dos solos de várzea, a dos Planossolos apresenta a maior extensão (56,6\%), ocupando cerca de $11 \%$ da área total do Estado. Os Planossolos apresentam um horizonte B plânico com cores acinzentadas ou escurecidas, indicativas de baixa permeabilidade, com incremento de argila em relação ao horizonte A ou E suficiente para caracterizar mudança textural abrupta (PINTO et al., 2004).

Diferentes indicadores físicos têm sido utilizados para avaliar a qualidade estrutural dos solos de várzea, como a densidade e porosidade do solo (PEDROTTI et al., 2001), a resistência do solo ao penetrômetro (LIMA et al., 1999) e a estabilidade de agregados (LIMA et al., 2003). A estabilidade de agregados do solo é resultado de processos biológicos, químicos e físicos que ocorrem interativamente no solo (TISDALL \& OADES, 1982). A partir do seu conhecimento, pode-se inferir sobre a capacidade de infiltração e retenção de água, condutividade hidráulica e erodibilidade do solo (LE BISSONNAIS, 1996). MÁRQUEZ et al. (2003) postulam que a qualidade do solo pode ser avaliada por meio da quantificação de agregados estáveis.

Dentre os fatores que influenciam a formação e a estabilização dos agregados, destacamse a textura (REICHERT et al., 1993), a mineralogia da fração argila, o tipo e teor de cátions presentes, a matéria orgânica e os tipos de microrganismos (REICHERT \& NORTON, 1994; CARTER, 2002), as raízes de plantas e resíduos vegetais (SILVA \& MIELNICZUK, 1998) e sistema de manejo do solo (LIMA et al., 2003).

A sistematização do solo compreende a adequação da superfície do terreno, envolvendo intenso tráfego de máquinas agrícolas para remover o solo (corte) e depositá-lo em outro local de cota inferior (aterro). Essa prática tem sido adotada com freqüência no estado do RS, que apresenta aproximadamente $13 \%$ de sua área orizícola sistematizada. Na região da Depressão Central do RS, 32\% da área orizícola encontra-se sistematizada, sendo que, em alguns municípios, como São João do Polêsine, 90\% da área orizícola é sistematizada (IRGA, 2004). A sistematização do terreno propicia menor consumo de água e maior eficiência no uso de máquinas agrícolas, favorecendo a aplicação de insumos e a introdução de outras culturas que compõem o sistema de cultivo juntamente com o arroz (PARFITT et al., 2004).

Em uma área sistematizada, nas áreas de corte, pode ocorrer a exposição do horizonte B, que geralmente é extremamente duro quando seco, muito firme quando úmido e muito plástico e muito pegajoso quando molhado. Nesses locais, são utilizadas máquinas agrícolas com potência e custo maiores para o preparo do solo. Caso apenas parte do horizonte superficial seja removido, o horizonte B fica próximo da superfície e poderá restringir o estabelecimento e o desenvolvimento de algumas culturas (SOSBAI, 2003).

Em solos do oeste de Arkansas, BRYE et al. (2003) observaram que o nivelamento ou a sistematização do terreno pode afetar a relação entre as propriedades físicas (densidade e textura) e as biológicas (biomassa bacteriana e fúngica). BRYE et al. (2004) discutem os efeitos da sistematização, em áreas de corte, sobre a variabilidade e a distribuição espacial das características químicas do solo. Os autores observaram um incremento significativo nos teores de $\mathrm{P}, \mathrm{K}, \mathrm{Mg}$ e Na e diminuição do $\mathrm{pH}$, da matéria orgânica e de Fe na camada de 0,00 a $0,10 \mathrm{~m}$.

No Brasil, há carência de informações sobre os efeitos da sistematização sobre as propriedades do solo, em especial sobre a agregação. A hipótese testada neste estudo é a de que a sistematização altera a estabilidade de agregados dos horizontes de perfis de solos de várzea. Assim, o objetivo deste trabalho foi avaliar a influência das propriedades físicas e químicas sobre o grau de agregação e verificar a semelhança de características relacionadas à agregação do solo de perfis de solos originais e sistematizados.

\section{MATERIAL E MÉTODOS}

O estudo foi conduzido nos anos de 1996 e 1997, numa área situada no município de São João do Polêsine, RS (29³5'08” e 2941'55' S; 5332'08” e 5322'56” O), em perfis de solo onde ocorre um Planossolo Hidromórfico eutrófico típico (EMBRAPA, 1999), em duas condições: alterado pela sistematização e original. O clima da região é caracterizado como Cfa, de acordo com a classificação de Köppen. A precipitação pluvial média anual é de $1600 \mathrm{~mm}$, com chuvas concentradas nos meses de abril, maio, setembro e outubro. A temperatura média anual mínima encontra-se na faixa de 4 a $15^{\circ} \mathrm{C}$ e a máxima entre 23 a $25^{\circ} \mathrm{C}$. Foram estudados seis perfis, sendo dois em áreas originais, sem sistematização, dois em área sistematizada, no aterro $(0-0,05 \mathrm{~m}$ e $0,05-0,10 \mathrm{~m})$ e dois em área sistematizada, no corte (0 - 0,05m e 0,05-0,10m). As características morfológicas dos perfis, 0 procedimento de análise e os resultados das determinações químicas e físicas são encontrados em NUNES et al. (2002). O ferro, o alumínio e o manganês total $\left(\mathrm{Fe}_{\mathrm{t}}, \mathrm{Al}_{\mathrm{t}}, \mathrm{Mn}_{\mathrm{t}}\right)$ foram quantificados conforme DICK \& KÄMPF (1988). Os mesmos elementos foram extraídos com ditionito-citrato-bicarbonato $\left(\mathrm{Fe}_{\mathrm{d}}, \mathrm{Al}_{\mathrm{d}}, \mathrm{Mn}_{\mathrm{d}}\right)$ (MEHRA \& JACKSON, 1960) e com solução de oxalato de amônio $\left(\mathrm{Fe}_{0}, \mathrm{Al}_{0}, \mathrm{Mn}_{0}\right)$ (MCKEAGUE \& DAY, 1966). 
Para avaliação do grau de agregação do solo, amostras com estrutura preservada foram coletadas utilizando cilindros de Uhland. Após serem manualmente separados, os agregados foram secados ao ar. Foram empregados dois métodos: (i) método padrão (KEMPER \& CHEPIL, 1965), que utiliza agregados que passaram por peneira de $8 \mathrm{~mm}$ e ficaram retidos na peneira de malha de $4,76 \mathrm{~mm}$, e (ii) método modificado, que utiliza agregados que passaram pela peneira de $8 \mathrm{~mm}$. No peneiramento úmido, utilizaram-se peneiras de malha 4,$76 ; 2,00 ; 1,00$; e $0,21 \mathrm{~mm}$, para amostras do método padrão, obtendo-se o diâmetro médio ponderado $\left(\mathrm{DMP}_{\mathrm{PU}}\right)$ e geométrico $\left(\mathrm{DMG}_{\mathrm{PU}}\right)$ dos agregados do solo. Para as amostras do método padrão modificado, obteve-se o diâmetro médio ponderado $\left(\mathrm{DMP}_{\mathrm{MU}}\right)$ e geométrico $\left(\mathrm{DMG}_{\mathrm{MU}}\right)$ dos agregados do solo. No peneiramento seco utilizaram-se amostras do método padrão modificado e peneiras de malha 4,76; 2,00; 1,00; 0,21 e 0,105mm, obtendo-se o diâmetro médio ponderado $\left(\mathrm{DMP}_{\mathrm{S}}\right)$ e geométrico $\left(\mathrm{DMG}_{\mathrm{S}}\right)$ dos agregados do solo. O diâmetro médio ponderado (DMP) e diâmetro médio geométrico (DMG) foram obtidos, respectivamente, pelas equações:

$$
D M P=\left[\frac{\sum_{i=1}^{n} D M i \times(M A G R \dot{t}-m i)}{\sum_{i=1}^{n}(M t A G R \dot{t}-m i t)}\right]
$$

e

$$
D M G=\exp \left[\frac{\sum_{i=1}^{n} \ln D M i \times(M A G R i-m i)}{\sum_{i=1}^{n}(M t A G R i-m i t)}\right]
$$

Sendo: $D M P=$ diâmetro médio ponderado dos agregados do solo; $D M G=$ diâmetro médio geométrico dos agregados do solo; $D M i=$ diâmetro médio de agregados do solo da classe i (mm); MAGRi, $M t A G R i=$ massa e massa total de agregados do solo da classe i (g); $m i$, mit= massa e massa total de material inerte da classe i (g). O índice de estabilidade ponderado (IEP) e o índice de estabilidade geométrico (IEG) foram, respectivamente, obtidos por:

$$
I E P=\frac{D M P_{P U}}{D M P_{S}} \quad \text { e } \quad I E G=\frac{D M G_{P U}}{D M G_{S}}
$$

Contrastes ortogonais foram feitos para avaliar a similaridade das camadas de perfil do solo sistematizado com horizontes do perfil original quanto à estabilidade de agregados. Efetuaram-se análises de correlação e de regressão linear simples e múltipla “stepwise” para quantificar a relação existente entre propriedades físicas e químicas e a estabilidade de agregados do solo.

\section{RESULTADOS E DISCUSSÃO}

Variação da agregação do solo no perfil e na área sistematizada

O maior tamanho médio de agregados a seco $\left(\mathrm{DMP}_{\mathrm{s}}\right.$ e $\mathrm{DMG}_{\mathrm{s}}$ ) no horizonte B (Tabela 1) pode estar relacionado aos maiores teores de argila $\left(417 \mathrm{~g} \mathrm{~kg}^{-1}\right)$, de ferro total $\left(19,5 \mathrm{~g} \mathrm{~kg}^{-1}\right)$, dos óxidos de ferro cristalinos $\left(11,0 \mathrm{~g} \mathrm{~kg}^{-1}\right)$ e de baixa cristalinidade $\left(9,1 \mathrm{~g} \mathrm{~kg}^{-1}\right)$ do perfil original, apresentados em NUNES et al. (2002). Os maiores valores de índices de estabilidade de agregados com peneiramento úmido $\left(\mathrm{DMP}_{\mathrm{PU}}, \mathrm{DMG}_{\mathrm{PU}}\right.$, $\mathrm{DMP}_{\mathrm{MU}}, \mathrm{DMG}_{\mathrm{MU}}$, IEP, IEG) (Tabela 1), no horizonte superficial, estão possivelmente relacionados ao maior teor de carbono orgânico. Apesar de não ser evidenciada uma correlação entre o teor de carbono orgânico e a estabilização de agregados (Tabela 2), os compostos orgânicos participam das ligações entre partículas individuais do solo. TISDALL \& OADES (1982) explicam que a correlação entre matéria orgânica e agregação nem sempre é observada pelo motivo de que somente parte da matéria orgânica é responsável pela estabilização dos agregados. Além disso, nem sempre os compostos orgânicos são os principais agentes cimentantes e principais atuantes no processo de estabilização dos agregados do solo.

A análise de contrastes ortogonais mostrou que o $\mathrm{DMP}_{\mathrm{S}}$ e o $\mathrm{DMG}_{\mathrm{S}}$ das áreas de corte e de aterro não coincidiram com os do horizonte $\mathrm{B}$ do perfil original. O solo movimentado, contudo, teve estabilidade similar à dos horizontes A e E do perfil original, correspondendo a uma profundidade de mobilização inferior a 0,70 m (Tabela 1). A agregação avaliada através do $\mathrm{DMP}_{\mathrm{PU}}, \mathrm{DMG}_{\mathrm{PU}}, \mathrm{DMP}_{\mathrm{MU}}, \mathrm{DMG}_{\mathrm{MU}}$, IEP e IEG dos perfis de corte e aterro foi similar à agregação dos horizontes $\mathrm{E}$ e $\mathrm{B}$ do perfil original. A exposição do horizonte $\mathrm{B}$ nessas áreas, que apresentam estrutura predominantemente prismática e consistência dura, firme e plástica e pegajosa, pode apresentar condições físicas desfavoráveis para o estabelecimento e crescimento das plantas. Esses fatores, associados ao elevado teor de argila e baixo teor de matéria orgânica, podem originar condições físicas limitantes à produtividade das culturas nos primeiros anos após a sistematização do solo (NUNES et al., 2002). Teores elevados de argila e a formação de crostas em horizontes subsuperficiais expostos poderão restringir o estabelecimento e o desenvolvimento de algumas culturas (BRYE et al., 2003). Após a remoção da 
Tabela 1 - Médias de $\mathrm{DMP}_{\mathrm{S}}, \mathrm{DMG}_{\mathrm{S}}, \mathrm{DMP}_{\mathrm{PU}}, \mathrm{DMG}_{\mathrm{PU}}, \mathrm{DMP}_{\mathrm{MU}}, \mathrm{DMG}_{\mathrm{MU}}$, IEP, IEG nos diferentes horizontes e/ou camadas de um Planossolo hidromórfico eutrófico típico sistematizado e sob cultivo de arroz irrigado

\begin{tabular}{|c|c|c|c|c|c|c|c|c|}
\hline & \multicolumn{8}{|c|}{ Método ${ }^{(1)}$} \\
\hline & $\mathrm{DMP}_{\mathrm{S}}$ & $\mathrm{DMG}_{\mathrm{S}}$ & $\mathrm{DMP}_{\mathrm{PU}}$ & $\mathrm{DMG}_{\mathrm{PU}}$ & $\mathrm{DMP}_{\mathrm{MU}}$ & $\mathrm{DMG}_{\mathrm{MU}}$ & IEP & IEG \\
\hline & \multicolumn{8}{|c|}{$\mathrm{mm}$} \\
\hline A & 2,61 & 1,43 & 4,98 & 3,37 & 2,17 & 0,84 & 0,85 & 0,62 \\
\hline E & 2,98 & 1,37 & 0,18 & 0,12 & 0,46 & 0,17 & 0,15 & 0,13 \\
\hline Btg & 4,43 & 3,29 & 1,88 & 0,58 & 0,56 & 0,27 & 0,13 & 0,08 \\
\hline Aterro $0,00-0,05 \mathrm{~m}$ & 2,37 & 1,17 & 0,42 & 0,20 & 0,33 & 0,15 & 0,14 & 0,13 \\
\hline Aterro $0,05-0,10 \mathrm{~m}$ & 2,82 & 1,74 & 0,63 & 0,26 & 0,49 & 0,20 & 0,18 & 0,12 \\
\hline Corte $0,00-0,05 \mathrm{~m}$ & 2,45 & 1,36 & 0,51 & 0,20 & 0,32 & 0,14 & 0,15 & 0,12 \\
\hline Corte $0,05-0,10 \mathrm{~m}$ & 3,48 & 2,28 & 0,72 & 0,26 & 0,40 & 0,19 & 0,11 & 0,08 \\
\hline \multicolumn{9}{|l|}{ Contrastes } \\
\hline A $x$ Btg & NS & NS & $* * *$ & $* * *$ & $* * *$ & $* * *$ & $* * *$ & $* * *$ \\
\hline Corte $(0,00-0,10 \mathrm{~m}) \times$ Btg & $* *$ & $* * *$ & NS & $* *$ & NS & NS & NS & NS \\
\hline Corte $(0,00-0,10 \mathrm{~m}) \times \mathrm{E}$ & NS & NS & NS & NS & NS & NS & NS & NS \\
\hline Corte $(0,00-0,10 \mathrm{~m}) \times \mathrm{A}$ & NS & NS & $* * *$ & $* * *$ & $* * *$ & $* * *$ & $* * *$ & $* * *$ \\
\hline Aterro $(0,00-0,10 \mathrm{~m}) \times \mathrm{A}$ & NS & NS & $* * *$ & $* * *$ & $* * *$ & $* * *$ & $* * *$ & $* * *$ \\
\hline Aterro $(0,00-0,10 \mathrm{~m}) \times \mathrm{E}$ & NS & NS & NS & NS & NS & NS & NS & NS \\
\hline Aterro $(0,00-0,10 \mathrm{~m}) \times \mathrm{Btg}$ & $* * *$ & $* * *$ & NS & $* *$ & NS & NS & NS & NS \\
\hline Aterro $(0,00-0,10 \mathrm{~m}) \times$ corte & $* * *$ & $* * *$ & NS & NS & NS & NS & NS & NS \\
\hline Aterro $0,05 \times 0,10 \mathrm{~m}$ & NS & NS & NS & NS & NS & NS & NS & NS \\
\hline Corte $0,05 \times 0,10 \mathrm{~m}$ & NS & NS & NS & NS & NS & NS & NS & NS \\
\hline
\end{tabular}

${ }^{(1)} \mathrm{DMP}_{\mathrm{S}}$ e $\mathrm{DMG}_{\mathrm{S}}=$ diâmetro médio ponderado e diâmetro médio geométrico obtidos por peneiramento seco; $\mathrm{DMP} \mathrm{Pu}_{\mathrm{PU}}$ e $\mathrm{DMG}$ : : diâmetro médio ponderado e diâmetro médio geométrico obtidos pelo método padrão e peneiramento úmido, $\mathrm{DMP}_{\mathrm{MU}}$ e $\mathrm{DMG}_{\mathrm{MU}}=$ diâmetro médio ponderado e diâmetro médio geométrico obtidos pelo método modificado e peneiramento úmido; IEP e IEG = índice de estabilidade ponderado e diâmetro médio geométrico dos agregados.

NS, **, *** = não significativo; significativo a $\mathrm{P}<0,01$ e significativo a $\mathrm{P}<0,001$, respectivamente.

superfície do solo pela sistematização ou nivelamento, o fato de as propriedades físicas dos horizontes subsuperficiais que ficaram expostos poderem permanecer relativamente inalteradas por aproximadamente 16 anos, podem acabar dificultando a restauração do potencial produtivo do solo, conforme observado por ECK (1987). Além disso, para efetuar o preparo do solo nessas áreas, serão necessárias máquinas agrícolas com maior potência e custo.

Correlação e regressão entre agregação e características e parâmetros do solo

Textura, densidade e porosidade do soloA argila correlacionou-se positivamente com o DMPs $\mathrm{e}$ o DMGs (Tabela 2), comprovando seu efeito na formação e estabilidade dos agregados do solo. A argila dispersa em água correlacionou-se positivamente com a agregação do solo $\left(\mathrm{DMP}_{\mathrm{S}}, \mathrm{DMG}_{\mathrm{S}}, \mathrm{DMP}_{\mathrm{PU}}\right.$ e $\left.\mathrm{DMG}_{\mathrm{PU}}\right)$, corroborando os resultados de REICHERT et al. (1993). A areia grossa correlacionou-se de forma positiva (DMG $_{\mathrm{PU}}, \mathrm{DMP}_{\mathrm{MU}}$, IEP e IEG) e negativa com a agregação do solo $\left(\mathrm{DMP}_{\mathrm{S}}\right.$ e $\left.\mathrm{DMG}_{\mathrm{S}}\right)$. A areia fina apresentou correlação negativa com o $\mathrm{DMP}_{\mathrm{s}}$ e o DMGs. A correlação negativa entre a areia grossa e a areia fina e a agregação do solo corrobora os resultados apresentados por REICHERT et al. (1993). A densidade e a porosidade total do solo não apresentaram correlação significativa com a agregação.

Na análise de regressão, observou-se que o índice de estabilidade de agregados (IEG), que representa a medida da agregação total do solo não considerando a distribuição por classe de tamanho de agregados (KEMPER \& CHEPIL, 1965), também esteve relacionado significativa e positivamente com a argila $\left(\mathrm{R}^{2}=0,51\right)$ (Tabela 3).

Óxidos de ferro - Os óxidos de ferro correlacionaram-se positivamente com o $\mathrm{DMP}_{\mathrm{S}}$ e o $\mathrm{DMG}_{\mathrm{s}}$ (Tabela 2). Os óxidos de ferro, devido a sua grande reatividade e elevada área superficial específica, são considerados agentes cimentantes das partículas primárias. De acordo com SCHWERTMANN \& TAYLOR (1989), o efeito da agregação provocada por óxidos de ferro varia de acordo com a quantidade e o tipo deste mineral, sendo que o principal mecanismo de agregação é a atração das cargas positivas das partículas de óxidos de ferro e pelas cargas negativas das argilas silicatadas. Observou-se que, dentre as formas de óxidos de ferro presentes nos solos de 
Tabela 2 - Coeficientes de correlação linear simples entre o diâmetro médio ponderado (DMP $)_{\mathrm{S}}$ e o diâmetro médio geométrico (DMG $)$ obtidos por peneiramento seco, diâmetro médio ponderado ( $\mathrm{DMP}_{\mathrm{PU}}$ ) e diâmetro médio geométrico (DMG $\mathrm{DP}_{\mathrm{PU}}$ obtidos pelo método padrão e peneiramento úmido, diâmetro médio ponderado $\left(\mathrm{DMP}_{\mathrm{MU}}\right)$ e diâmetro médio geométrico $\left(\mathrm{DMG} \mathrm{G}_{\mathrm{MU}}\right)$ obtidos pelo método modificado e peneiramento úmido, índice de estabilidade ponderado (IEP) e índice de estabilidade geométrico (IEG) dos agregados e propriedades de um Planossolo Hidromórfico eutrófico típico sistematizado e sob cultivo de arroz irrigado

\begin{tabular}{|c|c|c|c|c|c|c|c|c|}
\hline $\begin{array}{l}\text { Propriedades } \\
\text { intrínsecas do solo } \\
(1)\end{array}$ & $\mathrm{DMP}_{\mathrm{S}}$ & $\mathrm{DMG}_{\mathrm{S}}$ & $\mathrm{DMP}_{\mathrm{PU}}$ & $\mathrm{DMG}_{\mathrm{PU}}$ & $\mathrm{DMP}_{\mathrm{MU}}$ & $\mathrm{DMG}_{\mathrm{MU}}$ & IEP & IEG \\
\hline$A_{R G}$ & $0,71^{* *}$ & $0,77^{* *}$ & $-0,09$ & $-0,24$ & $-0,27$ & $-0,22$ & $-0,36$ & $-0,40$ \\
\hline$A_{D A}$ & $0,67 *$ & $0,78^{* *}$ & $0,61^{*}$ & $0,69 *$ & 0,21 & 0,53 & $-0,42$ & $-0,70^{*}$ \\
\hline S/A & $-0,62 *$ & $-0,74^{* *}$ & 0,05 & 0,20 & 0,25 & 0,19 & 0,33 & 0,37 \\
\hline$A_{G}$ & $-0,76 * * *$ & $-0,74 * *$ & 0,40 & $0,55^{*}$ & $0,55^{*}$ & 0,51 & $0,64^{*}$ & $0,67 * *$ \\
\hline $\mathrm{A}_{\mathrm{F}}$ & $-0,66 * *$ & $-0,71^{* *}$ & 0,09 & 0,22 & 0,26 & 0,21 & 0,34 & 0,38 \\
\hline $\mathrm{S}$ & $-0,44$ & $-0,56^{*}$ & $-0,16$ & $-0,05$ & $-0,01$ & $-0,06$ & 0,06 & 0,09 \\
\hline $\mathrm{D}_{\mathrm{S}}$ & 0,08 & $-0,06$ & 0,23 & 0,30 & 0,35 & 0,33 & 0,32 & 0,30 \\
\hline $\mathrm{P}_{\mathrm{T}}$ & 0,01 & 0,15 & $-0,28$ & $-0,36$ & $-0,42$ & $-0,39$ & $-0,39$ & $-0,38$ \\
\hline $\mathrm{Fe}_{\mathrm{t}}$ & $0,77^{* *}$ & $0,84^{* * *}$ & 0,07 & $-0,08$ & $-0,12$ & $-0,06$ & $-0,23$ & $-0,27$ \\
\hline $\mathrm{Fe}_{\mathrm{d}}$ & $0,76^{* *}$ & $0,82 * * *$ & $-0,03$ & $-0,19$ & $-0,22$ & $-0,16$ & $-0,32$ & $-0,36$ \\
\hline $\mathrm{Fe}_{\mathrm{o}}$ & $0,84 * * *$ & $0,89 * * *$ & 0,08 & $-0,11$ & $-0,14$ & $-0,08$ & $-0,26$ & $-0,30$ \\
\hline $\mathrm{Fe}_{\mathrm{d}} / \mathrm{Fe}_{\mathrm{t}}$ & $-0,05$ & $-0,25$ & $-0,58 *$ & $-0,51$ & $-0,43$ & $-0,47$ & $-0,42$ & $-0,40$ \\
\hline $\mathrm{Fe}_{\mathrm{o}} / \mathrm{Fe}_{\mathrm{d}}$ & 0,53 & $0,54^{*}$ & 0,01 & $-0,13$ & $-0,15$ & $-0,11$ & $-0,22$ & $-0,23$ \\
\hline $\mathrm{Al}_{\mathrm{t}}$ & $0,82 * * *$ & $0,88 * * *$ & 0,04 & $-0,12$ & $-0,17$ & $-0,10$ & $-0,28$ & $-0,32$ \\
\hline $\mathrm{Al}_{\mathrm{d}}$ & $0,64^{*}$ & $0,75^{* *}$ & $-0,15$ & $-0,30$ & $-0,35$ & $-0,29$ & $-0,43$ & $-0,47$ \\
\hline $\mathrm{Al}_{0}$ & 0,52 & $0,65^{*}$ & $-0,24$ & $-0,38$ & $-0,43$ & $-0,37$ & $-0,48$ & $-0,52$ \\
\hline $\mathrm{Al}_{\mathrm{d}} / \mathrm{Al}_{\mathrm{t}}$ & $-0,58^{*}$ & $-0,47$ & $-0,46$ & $-0,40$ & $-0,43$ & $-0,44$ & $-0,32$ & $-0,29$ \\
\hline $\mathrm{Al}_{\mathrm{o}} / \mathrm{Al}_{\mathrm{d}}$ & $-0,16$ & 0,01 & $-0,25$ & $-0,28$ & $-0,32$ & $-0,30$ & $-0,29$ & $-0,30$ \\
\hline $\mathrm{Mn}_{\mathrm{t}}$ & $-0,16$ & $-0,33$ & 0,38 & 0,47 & 0,53 & 0,48 & 0,52 & $0,54 *$ \\
\hline $\mathrm{Mn}_{\mathrm{d}}$ & $-0,42$ & $-0,53$ & $0,57 *$ & $0,69 * *$ & $0,73^{* *}$ & $0,69 * *$ & $0,76^{* *}$ & $0,78^{* *}$ \\
\hline $\mathrm{Mn}_{\mathrm{d}} / \mathrm{Mn}_{\mathrm{t}}$ & $-0,70 * *$ & $-0,58 *$ & $-0,19$ & $-0,10$ & $-0,14$ & $-0,16$ & $-0,02$ & 0,01 \\
\hline $\mathrm{Ca}$ & 0,48 & $0,56^{*}$ & $-0,22$ & $-0,35$ & $-0,41$ & $-0,36$ & $-0,44$ & $-0,46$ \\
\hline $\mathrm{Mg}$ & $0,76^{* *}$ & $0,80 * * *$ & 0,001 & $-0,15$ & $-0,19$ & $-0,13$ & $-0,28$ & $-0,32$ \\
\hline $\mathrm{K}$ & $-0,02$ & 0,10 & $-0,43$ & $-0,47$ & $-0,53$ & $-0,51$ & $-0,49$ & $-0,49$ \\
\hline $\mathrm{Na}$ & $0,79 * * *$ & $0,83^{* * *}$ & $-0,08$ & $-0,23$ & $-0,27$ & $-0,21$ & $-0,36$ & $-0,40$ \\
\hline SB & $0,57 *$ & $0,65^{*}$ & $-0,17$ & $-0,32$ & $-0,37$ & $-0,32$ & $-0,43$ & $-0,45$ \\
\hline $\mathrm{Al}$ & $0,82 * * *$ & $0,90 * * *$ & 0,02 & $-0,17$ & $-0,21$ & $-0,14$ & $-0,32$ & $-0,36$ \\
\hline $\mathrm{H}+\mathrm{Al}$ & 0,42 & 0,58 & $-0,06$ & $-0,22$ & $-0,29$ & $-0,23$ & $-0,31$ & $-0,35$ \\
\hline CTC & 0,54 & 0,67 & $-0,12$ & $-0,29$ & $-0,36$ & $-0,30$ & $-0,40$ & $-0,44$ \\
\hline V & 0,11 & $-0,10$ & $-0,12$ & $-0,05$ & 0,01 & $-0,02$ & $-0,02$ & 0,002 \\
\hline $\mathrm{C}_{\mathrm{ORG}}$ & $-0,44$ & $-0,29$ & 0,24 & 0,28 & 0,25 & 0,25 & 0,30 & 0,30 \\
\hline
\end{tabular}

(1) $\mathrm{A}_{\mathrm{RG}}=$ argila; $\mathrm{A}_{\mathrm{DA}}=$ argila dispersa em água; $\mathrm{S} / \mathrm{A}=$ silte/argila; $\mathrm{A}_{\mathrm{G}}=$ areia grossa; $\mathrm{A}_{\mathrm{F}}=$ areia fina; $\mathrm{D}_{\mathrm{S}}=$ densidade do solo; $\mathrm{P}_{\mathrm{T}}=$ porosidade total; $\mathrm{Fe}_{\mathrm{t}}=$ ferro total; $\mathrm{Fe}_{\mathrm{o}}=$ óxido de ferro extraído com solução de ditionito-citrato-bicarbonato; $\mathrm{Fe}_{\mathrm{o}}=$ óxido de ferro extraído com solução de oxalato de amônio; $\mathrm{Al}_{\mathrm{t}}=$ alumínio total; $\mathrm{Al}_{\mathrm{d}}=$ óxido de alumínio extraído com solução de ditionito-citrato-bicarbonato; $\mathrm{Al}_{\mathrm{o}}=$ óxido de alumínio extraído com solução de oxalato de amônio; $\mathrm{Mn}_{\mathrm{t}}=$ manganês total; $\mathrm{Mn}_{\mathrm{d}}=$ óxido de manganês extraído com solução de ditionitocitrato-bicarbonato e $\mathrm{C}_{\mathrm{ORG}}=$ carbono orgânico.

$*$, **, *** = significativo a $\mathrm{P}<0,05$; significativo a $\mathrm{P}<0,01$ e significativo a $\mathrm{P}<0,001$, respectivamente.

várzea, os de baixa cristalinidade $\left(\mathrm{Fe}_{\mathrm{o}}\right)$ foram responsáveis pela variação significativa e positiva dos valores de $\mathrm{DMP}_{\mathrm{S}}, \mathrm{DMG}_{\mathrm{s}}$, $\mathrm{DMP}_{\mathrm{PU}}, \mathrm{DMG}_{\mathrm{PU}}, \mathrm{DMG}_{\mathrm{MU}}$ (Tabela 3). A influência de diferentes formas de óxidos de ferro na agregação do solo foi mencionada por IMHOFF et al. (2002) e PEDROTTI et al. (2003). IMHOFF et al. (2002) verificaram uma positiva e significativa relação entre os óxidos de ferro de baixa cristalinidade $\left(\mathrm{Fe}_{0}\right)$ e a resistência tênsil dos agregados de solos com diferentes texturas. Similarmente, SILVA
\& MIELNICZUK (1998) evidenciaram uma relação positiva e significativa entre $\mathrm{Fe}_{0}$ e a agregação do solo. REICHERT et al. (1993) obtiveram correlação significativa e positiva entre $\mathrm{DMP}_{\mathrm{S}}$ e óxidos de ferro de baixa cristalinidade. A maior capacidade de agregação condicionada à presença de óxidos de ferro de baixa cristalinidade atribui-se, segundo DUIKER et al. (2003), à maior área superficial reativa que esses compostos possuem em relação à apresentada por óxidos de ferro cristalinos.

Ciência Rural, v.36, n3, mai-jun, 2006. 
Tabela 3 - Regressão linear múltipla entre o grau de agregação e propriedades intrínsecas de um Planossolo hidromórfico eutrófico típico sistematizado e sob cultivo de arroz irrigado.

\begin{tabular}{lll}
\hline $\begin{array}{l}\text { Variáveis } \\
\text { dependentes }\end{array}$ & $\begin{array}{l}\text { Parâmetros estimados e } \\
\text { variáveis independentes }\end{array}$ & $\mathrm{R}^{2}$ \\
\hline $\mathrm{DMP}_{\mathrm{S}}$ & $4,122-0,021 \mathrm{~A}_{\mathrm{G}}+0,136 \mathrm{Fe}_{\mathrm{o}}$ & $0,79 * * *$ \\
$\mathrm{DMG}_{\mathrm{S}}$ & $0,985+0,070 \mathrm{Fe}_{\mathrm{d}}+0,168 \mathrm{Fe}_{\mathrm{o}}$ & $0,85^{* * *}$ \\
$\mathrm{DMP}_{\mathrm{PU}}$ & $0,198+0,186 \mathrm{Fe}_{\mathrm{o}}$ & $0,66 * *$ \\
$\mathrm{DMG}_{\mathrm{PU}}$ & $-0,017+0,051 \mathrm{Fe}_{\mathrm{o}}+0,176 \mathrm{Al}_{\mathrm{o}} / \mathrm{Al}_{\mathrm{d}}$ & $0,92^{* * *}$ \\
$\mathrm{DMP}_{\mathrm{MU}}$ & $0,581+-0,385 \mathrm{Mn}_{\mathrm{d}} / \mathrm{Mn}_{\mathrm{t}}$ & $0,29 \mathrm{NS}$ \\
$\mathrm{DMG}_{\mathrm{MU}}$ & $0,149+0,013 \mathrm{Fe}_{\mathrm{o}}$ & $0,63^{* *}$ \\
$\mathrm{IEP}_{\mathrm{IEG}}$ & $0,069+0,0002 \mathrm{~A}_{\mathrm{F}}$ & $0,21 \mathrm{NS}$ \\
\hline
\end{tabular}

${ }^{(1)} \mathrm{DMP}_{\mathrm{S}}$ e $\mathrm{DMG}_{\mathrm{S}}$ = diâmetro médio ponderado e diâmetro médio geométrico obtidos por peneiramento via seco; $\mathrm{DMP}_{\mathrm{PU}}$ e $\mathrm{DMG}_{\mathrm{PU}}$ = diâmetro médio ponderado e diâmetro médio geométrico obtidos pelo método padrão e peneiramento via úmido, $\mathrm{DMP}_{\mathrm{MU}} \mathrm{e}$ $\mathrm{DMG}_{\mathrm{MU}}=$ diâmetro médio ponderado e diâmetro médio geométrico obtidos pelo método modificado e peneiramento via úmido; IEP e IEG = índice de estabilidade ponderado e índice de estabilidade geométrico dos agregados.

${ }^{(2)} \mathrm{A}_{\mathrm{G}}=$ areia grossa; $\mathrm{A}_{\mathrm{F}}=$ areia fina; $\mathrm{A}_{\mathrm{RG}}=$ argila; $\mathrm{Fe}_{\mathrm{d}}=$ óxido de ferro extraído com solução de ditionito-citrato-bicarbonato; $\mathrm{Fe}_{\mathrm{o}}=$ óxido de ferro extraído com solução de oxalato de amônio; $\mathrm{Al}_{\mathrm{d}}=$ óxido de alumínio extraído com solução de ditionito-citratobicarbonato; $\mathrm{Al}_{0}=$ óxido de alumínio extraído com solução de oxalato de amônio; $\mathrm{Mn}_{\mathrm{t}}=$ manganês total; $\mathrm{Mn}_{\mathrm{d}}=$ óxido de manganês extraído com solução de ditionito-citrato-bicarbonato $\mathrm{NS},{ }^{* *}, * * *$ = não significativo, significativo a $\mathrm{P}<0,01 \mathrm{e}$ significativo a $\mathrm{P}<0,001$, respectivamente.

Óxidos de alumínio e de manganês - $\mathrm{O}$ alumínio $\left(\mathrm{Al}_{\mathrm{d}}\right)$ e $\left(\mathrm{Al}_{\mathrm{t}}\right)$ teve correlação com o $\mathrm{DMP}_{\mathrm{s}}$ e o $\mathrm{DMG}_{\mathrm{s}}$, assim como o manganês $\left(\mathrm{Mn}_{\mathrm{d}}\right)$ também correlacionou-se com a agregação do solo ( $\mathrm{DMP}_{\mathrm{PU}}$, $\mathrm{DMG}_{\mathrm{Pu}}, \mathrm{DMP}_{\mathrm{MU}}, \mathrm{DMG}_{\mathrm{MU}}$, IEP e IEG) (Tabela 2). Óxidos de alumínio cristalinos $\left(\mathrm{Al}_{\mathrm{d}}\right)$ e de baixa cristalinidade $\left(\mathrm{Al}_{0}\right)$ relacionaram-se positivamente com o $\mathrm{DMG}_{\mathrm{PU}}\left(\mathrm{R}^{2}\right.$ $=0,92$ ) (Tabela 3). Dois mecanismos podem ser responsáveis pela agregação dos solos: (i) polímeros pequenos de alumínio tipo $\mathrm{Al}-\mathrm{OH}$ podem aderir-se mais fortemente nas entre camadas de argilominerais expansíveis do que cátions trocáveis, reduzindo a expansão dos argilominerais e promovendo a agregação do solo e (ii) polímeros de $\mathrm{Al}-\mathrm{OH}$ podem ligar-se à superfície externa de partículas de argila, formando agregados (HSU, 1989). PEDROTTI et al. (2003) observaram uma participação significativa e positiva de formas de alumínio amorfas e de baixa cristalinidade na agregação do solo, devido, segundo esses autores, à grande capacidade da matéria orgânica para complexar o alumínio. VITORINO et al. (2003) apresentaram a importância positiva das diferentes formas de alumínio na resistência do solo à desagregação e, por sua vez, nas propriedades relacionadas à agregação do próprio solo. No entanto, deve-se considerar que parte do $\mathrm{Al}_{0}$ e, principalmente, do $\mathrm{Al}_{\mathrm{d}}$ devem ser provenientes da substituição isomórfica em óxidos de ferro, corroborando os resultados de INDA JUNIOR \& KÄMPF (2003).

Cátions trocáveis e matéria orgânica - O cálcio trocável, apesar de apresentar características favoráveis à agregação do solo, não esteve geralmente correlacionado com os índices de agregação do solo (Tabela 2), concordando com os resultados obtidos por DUIKER et al. (2003). Similarmente, não foi encontrada correlação entre a agregação, a capacidade de troca de cátions (CTC) e a saturação de bases (V). Por outro lado, o magnésio, o sódio, a soma de bases trocáveis (SB) e o alumínio trocável correlacionaramse positivamente com DMPs e DMGs. Apesar de o sódio possuir característica dispersante, a correlação significativa pode ser explicada pelo motivo de que solos com alto teor desse elemento apresentam também uma maior concentração de outros cátions trocáveis (di e trivalentes), podendo esses atuarem mais fortemente na agregação do solo e, possivelmente, superando o efeito do Na. Analisando-se a possível correlação existente entre o $\mathrm{Na}$ e os demais elementos nesse estudo, observou-se uma correlação significativa com o Ca $(\mathrm{P}<0,0603)$, com o Mg $(\mathrm{P}<0,0008)$ e com o Al $(\mathrm{P}<0,0012)$. Não houve correlação significativa entre o carbono orgânico e a agregação do solo. Esses resultados estão de acordo com o trabalho de REICHERT \& NORTON (1994), mas discordam das conclusões obtidas por outros autores (PINHEIRO et al., 2004). HAYNES (2000) apresentou uma relação curvilinear entre a estabilidade dos agregados e o teor de carbono orgânico, evidenciando um aumento da agregação com o incremento da matéria orgânica do solo até aproximadamente $30 \mathrm{~g} \mathrm{~kg}^{-1}$, permanecendo constante a partir desse valor.

Considerando que a sistematização pode alterar a variabilidade espacial (vertical e horizontal) do solo, estudos envolvendo práticas de manejo que visem à melhoria ou à manutenção das propriedades físicas e, por sua vez, a condições adequadas ao estabelecimento e desenvolvimento das culturas, devem ser implementados em solos de várzea do RS. 


\section{CONCLUSÕES}

A agregação de camadas sistematizadas (corte e aterro) foi, em geral, similar à dos horizontes $\mathrm{E}$ e $\mathrm{B}$ do perfil original do solo. A argila, os óxidos de ferro, alumínio e manganês, além do magnésio e alumínio trocáveis, foram os principais componentes que se correlacionaram com a agregação do solo. Pela análise de regressão múltipla, verificou-se que houve relação positiva da agregação do solo com a argila e com os óxidos de ferro e de alumínio cristalinos e de baixa cristalinidade.

\section{REFERÊNCIAS}

AZAMBUJA, I.H.V. et al. Aspectos sócio econômicos da produção do arroz. In: GOMES, A. da S.; MAGALHÃES JÚNIOR, A.M. de. Arroz irrigado no sul do Brasil. Pelotas: Embrapa Clima Temperado, 2004. p.23-44.

BRYE, K.R. et al. Short-term effects of land leveling on soil physical properties and microbial biomass. Soil Science Society of America Journal, v.67, p.1405-1417, 2003.

BRYE, K.R. et al. Short-term effects of land leveling on soil chemical properties and their relationships with microbial biomass. Soil Science Society of American Journal, v.68, p.924-934, 2004.

CARTER, M.R. Soil quality for sustainable land management: Organic matter and aggregation interactions that maintain soil functions. Agronomy Journal, v.94, p.38-47, 2002.

DICK, D.P.; KÄMPF, N. Comparação da extração de ferro com $\mathrm{HCl} 6 \mathrm{~N}, \mathrm{H}_{2} \mathrm{SO}_{4}$ 1:1 e ditionito-citrato-bicarbonato de sódio em horizontes B latossólicos. Revisa Brasileira de Ciência do Solo, v.12, p.185-188, 1988.

DUIKER, S.W. et al. Iron (hidr)oxide crystallinity effects on soil aggregation. Soil Science Society of America Journal, v.67, p.606-611, 2003.

ECK, H.V. Characteristics of exposed subsoil-at exposure and 23 years later. Agronomy Journal, v.9, p.1067-1073, 1987.

EMBRAPA. Centro Nacional de Pesquisa de Solos. Sistema Brasileiro de Classificação de Solos. Brasília: Embrapa Produção de Informação; Rio de Janeiro: Embrapa Solos, 1999. 421p.

HAYNES, R.J. Interactions between soil organic matter status, cropping history, method of quantification and sample pretreatment and their effects on measured aggregate stability. Biology Fertility Soils, v.30, p.270-275, 2000.

HSU, P.H. Aluminum oxides and oxihidroxides. In: DIXON, J.B.; WEED, S.B. Minerals in soil environments. 2.ed. Madison: American Society of Agronomy: Soil Science Society of America, 1989. Cap.7, p.331-371.

INDA JUNIOR, A.V.; KÄMPF, N. Avaliacao de procedimentos de extração dos óxidos de ferro pedogênicos com ditionito citrate-bicarbonato de sódio. Revisa Brasileira de Ciência do Solo, v.27, p.1139-1147, 2003.

IMHOFF, S. et al. Factors contributing to the tensile strength and friability of Oxisols. Soil Science Society of America Journal, v.66, p.1656-1661, 2002.

IRGA. Instituto Rio Grandense do Arroz. Porto Alegre, RS. Capturado em 1 set. 2004. Online. Disponível na internet http://www.irga.rs.gov.br/arquivos/Areas_sistematizadas.pdf.

KEMPER, W.D.; CHEPIL, W.S. Size distribution of aggregates. In: BLACK, C.A. Methods of soil analysis: physical and mineralogical methods. Madison: American Society of Agronomy, 1965. p.499-509.

LE BISSONNAIS, Y. Aggregate stability and assessment of soil crustability and erodibility: I - Theory and methodology. European Journal of Soil Science, v.47, p.425-437, 1996.

LIMA, C.L.R. et al. Estabilidade de agregados de um Planossolo sob diferentes sistemas de manejo. Revista Brasileira de Ciência do Solo, v.27, p.199-205, 2003.

LIMA, C.L.R. et al. Resistência mecânica de um Planossolo cultivado com arroz irrigado sob diferentes sistemas de manejo. In: CONGRESSO BRASILEIRO DE ARROZ IRRIGADO, 23., 1999, Pelotas, RS. Anais... Pelotas: Embrapa Clima Temperado, 1999. p.253-256.

MÁRQUEZ, C.O. et al. Aggregate-size stability distribuition and soil stability. Soil Science Society of America Journal, v.68, p.725-735, 2003.

MCKEAGUE, J.A.; DAY, J.H. Dithionite and oxalateextractable $\mathrm{Fe}$ and $\mathrm{Al}$ as aids in differentiating various classes of soils. Canadian Journal of Soil Science, v.46, p.13-22, 1966.

MEHRA, O.P.; JACKSON, M.L. Iron oxide removal from soils and clays by a ditionite-citrate system buffered with sodium bicarbonate. Clays and Clay minerals, v.7, p.317-327, 1960.

NUNES, M.L. et al. Características de solos sistematizados em duas áreas cultivadas com arroz irrigado sob inundação. Revista Brasileira de Ciência do Solo, v.26, p.395-406, 2002.

PARFITTI, J.M.B. et al. Estruturação e sistematização da lavoura de arroz irrigado. In: GOMES, A. da S.; MAGALHÃES JÚNIOR, A.M. de. Arroz irrigado no Sul do Brasil. Pelotas: Embrapa Clima Temperado, 2004. p.237-257.

PEDROTTI, A. et al. Relação entre atributos físicos, mineralogia da fração argila e formas de alumínio no solo. Revista Brasileira de Ciência do Solo, v. 27, p.1-9, 2003.

PEDROTTI, A. et al. Sistemas de cultivo de arroz irrigado e a compactação de um Planossolo. Pesquisa Agropecuária Brasileira, p.36, p.709-715, 2001.

PINHEIRO, E.F.M. et al. Aggregate distribuition and soil organic matter under different tillage systems for vegetable crops in a Red Latosol from Brazil. Soil and Tillage Research, v.77, p.79-84, 2004. 
PINTO, L.F.S. et al. Solos de várzea do Sul do Brasil cultivados com arroz irrigado. In: GOMES, A. da S.; MAGALHÃES JÚNIOR. A.M. Arroz irrigado no sul do Brasil. Brasília: Embrapa Informação Tecnológica, 2004. p.75-95.

REICHERT, J.M.; NORTON, L.D. Aggregate stability and rain impacted sheet erosion of air dried and prewetted clayed surface soils under intense rain. Soil Science, v.158, p.159-169, 1994.

REICHERT, J.M. et al. Índices de estabilidade de agregados e suas relações com características e parâmetros do solo. Revista Brasileira de Ciência do Solo, v.17, p.283-290, 1993.

SCHWERTMAN, U.; TAYLOR, R.M. Iron oxides. In: DIXON, J.B.; WEED, S.B. Minerals in soil environments. 2.ed. Madison: American Society of Agronomy, Soil Science Society of America, 1989. p.379-427.
SILVA, I. de F.; MIELNICZUK, J. Sistemas de cultivo e características do solo afetando a estabilidade de agregados. Revista Brasileira de Ciência do Solo, v.22, p.311-317, 1998.

SOSBAI. Sociedade Sul Brasileira de Arroz Irrigado. Arroz irrigado: Recomendações técnicas da pesquisa para o Sul do Brasil. Itajaí, 2003. 126p.

TISDALL, J.M.; OADES, J.M. Organic matter and water stable aggregates in soil. Journal of Soil Science, v.33, p.141-163, 1982.

VITORINO, A.C.T. et al. Mineralogia, química e estabilidade de agregados do tamanho de silte de solos da região do sudeste do Brasil. Pesquisa Agropecuária Brasileira, v.38, p.133141, 2003. 